\title{
CuidArt: PROYECTO DE ARTE DEL DEPARTAMENTO DE SALUD DE DÉNIA
}

\section{cuidArt: Art Project of the Health Department of Dénia}

\author{
Alicia Ventura Bordes \\ Directora del Proyecto Cuidart. Hospital de \\ Dénia \\ aliciaventura@gcultural.com \\ Ángel Giménez Sierra \\ Director Gerente del Departamento de Salud de \\ Dénia \\ RAFAEl SALA Lopez \\ Director Medico del Departamento Salud de \\ Dénia \\ Dr. Miguel Ángel Climent Suñer \\ Jefe del Servicio de Psiquiatría. Hospital de \\ Dénia \\ Ana Moreno Cotarelo
}

Proyecto cuidArt. Hospital de Dénia

\author{
Dr. Antonio Valdivia Pérez \\ Responsable de Medicina Preventiva. Hospital \\ de Dénia \\ Miguel Peris Godoy \\ Director Asistencial del Departamento Salud \\ Dénia \\ Dr. Joan Manuel Gassent Blesa \\ Jefe del Servicio de Oncología. Hospital de \\ Dénia
}
Dr. Fernando Ramos Torre
Jefe del Servicio de Nefrología. Hospital de Dénia

ISABEL SOLER TARRADELLAS
Arteterapeuta

Recibido:14 de junio de 2011

Aceptado: 20 de julio de 2011

\section{Resumen:}

Con esta iniciativa pionera en España, desarrollada por parte de la empresa DKV Seguros, se busca que la presencia del arte llene el Hospital de Dénia y forme un entorno mas humanizado. Así, junto a la Colección DKV: Arte y Salud, se muestra un importante proyecto escultórico, así como la intervención pictórica mural llevada a cabo en el área de Pediatría y desarrollada por un reconocido colectivo de Urban Art. Además, el proyecto cuenta con una Sala de Exposiciones dentro del propio hospital, que bajo el leivmotiv de Arte y Salud, programará exposiciones anuales, de producción propia y también en colaboración con distintas entidades públicas y privadas. Todo este proyecto se completa con un programa de actividades para pacientes de las distintas áreas del hospital: hemodiálisis, quimioterapia, psiquiatría y pediatría. Durante la semana se desarrollan talleres de teatro, musicoterapia y arteterapia, así como diversas intervenciones murales efímeras mientras los pacientes están recibiendo sus tratamientos. Un programa de arte al servicio de la salud, donde la humanización del entorno, el dialogo entre las diferentes disciplinas del arte y la medicina, serán la base de desarrollo del proyecto.

Palabras clave: Humanización de la atención, Arte, Musicoterapia, Terapia con arte, Evaluación de procesos y resultados. 
Ventura, Alicia, Valdivia, Antonio. Gimenez, Angel. Peris, Miguel. Sala, Rafael. Moreno, Ana. Soler, Isabel. 2011. Cuidart: Proyecto de arte del departamento de salud de Dénia. Arte, individuo y Sociedad, Vol. 23, Núm. Especial, 165-180

\begin{abstract}
:
With this initiative, pioneer in Spain, developed by DKV Seguros, the hospital is imbued with art in order to make a more humanized environment. This way, with DKV Collection: Art and Health, an important sculpture project is held as well as the wall paintings in the pediatric area, developed by an important Urban Art group. Besides, the project has an Exhibition Room, which, under the slogan of Art and Health, will hold annual exhibitions of self production as well as exhibitions in collaboration with different public and private institutions. This whole project is completed with a program of activities for patients of different areas: hemodialysis, chemotherapy, psychiatry and pediatrics. During the week drama workshops, music therapy and art therapy activities are developed at the hospital, as well as ephemeral wall paintings while patients are receiving their treatments. It is an art program at the service of health, where the humanization of the environment, the dialogue between different art disciplines and medicine will be the foundation upon which this project will be developed.
\end{abstract}

Key words: Humanization of assistance, Art, Music therapy, Art therapy, Outcome and process assessment.

Sumario: 1.Colección DKV: Arte y Salud. 2.Esculturas: Armonización del espacio y la poética 3. Pinturas Murales: un diseño de ensoñación para Pediatría. 4. Sala de Exposiciones del proyecto cuidArt. 5. Arte en vivo.6. Talleres para pacientes. 6. Colaboraciones con la comarca y las universidades: un mundo de posibilidades al servicio del arte. 7. Medición de resultados y proyectos de investigación. Referencias.

\title{
1.Introducción
}

El proyecto desarrollado por el Hospital de Dénia de la mano de DKV Seguros es una iniciativa pionera en España que muestra una nueva visión terapéutica del arte.

DKV apuesta por aportar valor a la gestión de las prestaciones sanitarias públicas, ya que como empresa aseguradora especializada en salud y miembro del grupo líder en Europa en seguros médicos puede utilizar su conocimiento y experiencia para mejorar el nivel de calidad asistencial ofrecido a los ciudadanos.

El Hospital de Dénia abrió sus puertas a principios de 2009 para atender a una población de más de 200.000 usuarios, aunque en época estival la cifra puede alcanzar las 300.000 personas. Sus instalaciones ocupan $42.500 \mathrm{~m} 2$ y dispone de unas 280 camas. La construcción del complejo corrió a cargo de Marina Salud. Su gestión responde al modelo de hospital de colaboración publico-privado del Servicio Sanitario Público (Campoy, 2008).

En ese marco, Marina Salud, una empresa participada en un $65 \%$ por DKV Seguros y en un 35\% por Ribera Salud, grupo empresarial de gestión sanitaria propiedad de las cajas Bancaja y CAM, gestionan el hospital publico de Dénia (Alicante), cuya función es dar servicio al Departamento de Salud de Dénia en el marco de una concesión administrativa. 
Desde sus compromisos fundacionales el proyecto del Hospital de Dénia consideró su obligación de esforzarse por elevar el listón de la exigencia en todo lo relacionado con el servicio sanitario en el ámbito público. Dos focos de exigencia basados en dos frentes: por un lado la implicación activa en políticas y programas de prevención y mantenimiento de la salud y por otro la capacitación de las personas, equipamiento tecnológico y organización para abordar la enfermedad, y como resultado generación de nuevos conocimientos y su aplicación.

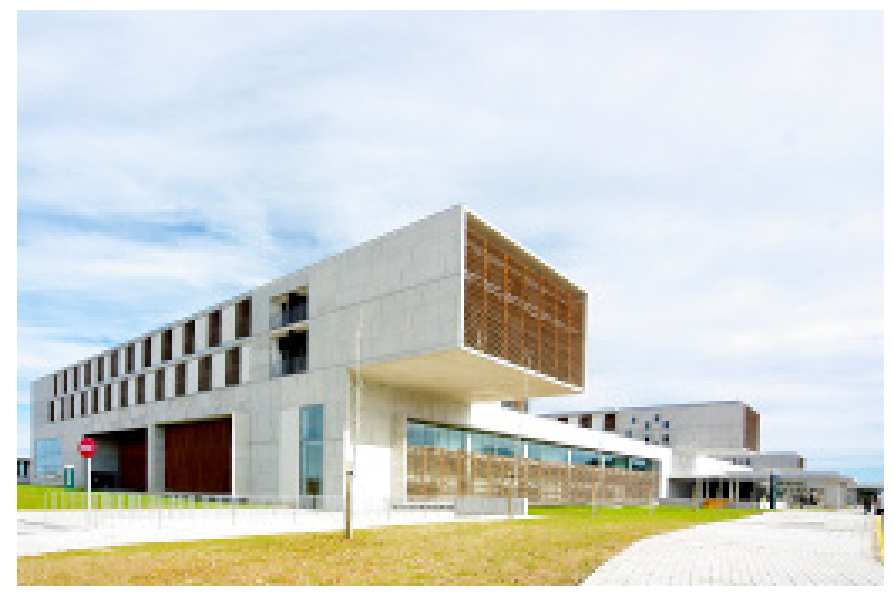

Fig.1 Hospital de Dénia.

El Hospital de Dénia se concibió arquitectónicamente como un espacio horizontal donde la luz juega un papel fundamental. Una arquitectura de signo humanizador, respetuosa con el paisaje y el medio ambiente, abierta a la luz mediterránea, coherente con los principios de un diseño que ayude a curar (Lawson, 2010).

La escenografía del Hospital fue concebida desde su primer plano como un lugar para albergar, dentro de un Hospital, un proyecto de Arte y Salud al servicio de los profesionales y los pacientes. Un proyecto destinado a ocuparse en primera instancia de la salud de las personas. Su conceptualización en términos de mediación simbólica ambiental (Belver y Ullán, 2010) permite identificar una integración de mediadores ambientales estructurales (arquitectura/construcción) y decorativos, estos últimos basados en el arte como elemento central, que producen una humanización del ambiente a través de la asociación con experiencias emocionales positivas.

En el Hospital entendemos la salud como un todo integral donde no puede desvincularse lo físico de lo anímico. Dentro del cultivo del bienestar emocional de las personas consideramos que el arte desempeña un papel fundamental. El arte alimenta el espíritu y forma parte de una concepción equilibrada de la naturaleza del ser humano. Humaniza los espacios (Belver y Ullán, 2010) y se ha convertido en una potente herramienta de ayuda para el tratamiento de algunas enfermedades.

El filósofo Emile M. Cioran explicó en numerosas ocasiones que su obra era fruto de un acto curativo, había comenzado a escribir para no volverse loco. El propio Dalí 
se definía como un paranoico a la vez que añadía: "Debo ser el único de mi especie que ha dominado y transformado en potencia creadora, gloria y júbilo una enfermedad mental tan grave".

Estos personajes han practicado, de algún modo, lo que ahora se denomina arteterapia, a la vez que han creado obras sublimes. Sin pretender pasar a la historia y ni siquiera con la intención de crear algo bello, el resto de los mortales puede emplear estas formas de expresión para superar alteraciones emocionales, como terapia de apoyo en trastornos psiquiátricos e incluso para hacer más llevaderas enfermedades tan graves como el cáncer o el alzheimer (Dileo, 2009). De hecho, Dalí no podría considerarse «el único» porque esta técnica lleva más de 50 años ayudando a pacientes de Gran Bretaña (Clift, 2009) y EEUU (Sonke, 2009). Ahora llega también a España.

Los artistas desembarcan en las organizaciones como fuente de creatividad e innovación. Asimismo, las empresas recurren a ellos no sólo como impulsores de cambios de mentalidad y de modernización, sino también para introducir nuevas estrategias de comunicación, recursos humanos y capacitación. El artista está ligado a su comunidad y busca nuevos caminos para integrarse en ella y aportar su creatividad en proyectos concretos, que mejoren la calidad de vida del conjunto de los ciudadanos o de colectivos concretos.

Con el fin de contribuir a la mejora de la salud y convertir el centro en un espacio más humano, el Hospital de Dénia comenzó a trabajar en este proyecto de manera paralela a la construcción de su edificio. Para ello, ha contado desde el inicio con el impulso de DKV Seguros, principal motor de este proyecto.

Así, con el Hospital de Dénia como escenario, el proyecto cuidArt aglutina diferentes disciplinas artísticas que engloba diferentes puestas en escena:

- La Colección DKV Arte y Salud: Un total de 28 destacados artistas han participado con sus obras en la colección DKV: Arte y Salud, uno de los pilares de este programa que la Aseguradora ha cedido temporalmente al Hospital.

- Un importante trabajo escultórico de siete artistas surgido en colaboración con el Instituto Arte y Entorno de la Universidad Politécnica de Valencia.

- Una intervención pictórica mural desarrollada en las diversas áreas de pediatría por un reconocido colectivo de Urban Art (Xlf crew).

- Una Sala de Exposiciones, que acoge un ambicioso programa bajo el epígrafe de la investigación en temas de Arte y Salud.

- Intervenciones de Arte en Vivo. Intervenciones artísticas en el Hospital de día Oncológico y de hemodiálisis con carácter efímero. Intervenciones artísticas en Psiquiatría realizada por los propios pacientes:

- Talleres para pacientes y personal sanitario: Arterapia y Musicoterapia. Teatro. Cuentacuentos, etc.

- Un plan de colaboraciones para ayudar a impulsar el arte a través de diferentes proyectos y escenarios: Colaboración con el Concurso de Lactancia Materna, exhibición fija de fotografías en maternidad. Producción del concurso Cuida't Vida Sana, itinerancia de exposiciones del Hospital que se trasladan al resto del Departamento de salud de la Marina Alta. Conciertos en colaboración con 
la Asociación de Bandas de Música de la Comunidad Valenciana. Y un largo etc. todavía en fase embrionaria

Manifestaciones, en fin que se pueden encontrar en cualquier momento y rincón del centro sanitario.

\section{Colección DKV: Arte y Salud}

La colección DKV Arte y Salud comenzó a gestarse de manera paralela a los planos del hospital con el fin de crear un entorno humanizado y saludable basado en las infinitas muestras históricas que ponen de manifiesto cómo numerosos enfermos han paliado su dolor a través del arte.

Una iniciativa impulsada por DKV Seguros médicos, que desde el inicio se implicó activamente con el Hospital en el desarrollo de programas de prevención y mantenimiento de la salud en la capacitación de personas, equipamiento tecnológico, y organización para abordar la enfermedad, las dolencias y discapacidades de los pacientes con la mayor expectativa de éxito. Esta colección responde al compromiso de la aseguradora por crear en el Hospital entornos que expresen el lado positivo de la vida y el fomento de actividades que alegren y enriquezcan este espacio.

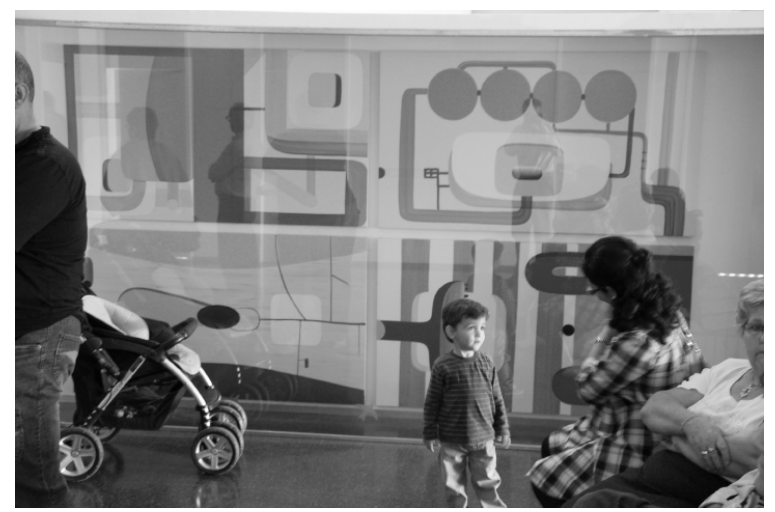

Fig.2 María Ortega. Sola, andando, contigo, en casa. Consultas externas de pediatría

Para montar esta colección, DKV Seguros ha nombrado a Alicia Ventura directora y coordinadora de este proyecto, que desde un primer momento ha contado con la colaboración de un Comité Asesor integrado por destacadas personalidades del mundo artístico: Juan Manuel Bonet, ex director de los museos IVAM y Reina Sofía; Juan Bautista Peiró, vicerrector de Cultura de la Universitat Politècnica de València; y Carlos Pérez, coordinador exposiciones del MUVIM.

El resultado, 28 obras pictóricas y fotográficas de destacados artistas de la Comunidad Valenciana de la generación de los 90 y del 2000 con un denominador común: han nacido o residen en la Comunidad valenciana: Sergio Barrera, Aurelio Ayela, María Cremades, Juan Cuéllar, Javier Garcerá, Moisés Mahiques, Regina de 
Miguel, Juan Olivares, Nico Munuera, María Ortega, Ramón Roig, Carolina Ferrer, Ernesto Casero, Chema López, Ximo Amigó, Toño Barreiro, Antonio Alcaraz, Roberto Mollá, Joël Mestre, Carlos Domingo, Javier Velasco, Nelo Vinuesa Sergio Belinchón, María Zarraga, Mira Bernabeu, Raúl Belinchón, Bleda y Rosa y Pilar Beltrán.

\section{Esculturas: Armonización del espacio y la poética}

El Hospital de Dénia también ha desarrollado un proyecto escultórico que busca la integración del edificio en el paisaje. Para ello, el Hospital ha trabajado en todo momento en colaboración con el Centro de Investigación Arte y Entorno de la Universidad Politécnica de Valencia (UPV), fundado en 1993.

Esta iniciativa parte, conceptualmente, de la integración entre el arte y la construcción con el fin de armonizar los espacios arquitectónicos creando "lugares" con significado. El propósito de este proyecto escultórico no es sólo el de crear objetos bellos sino el de construir obras que otorguen sentido a un momento vivido en el espacio del Hospital.

Los recursos formales y expresivos creados para cuidArt emanan de la singularidad poética de los artistas. Así, las obras en la mayoría de los casos, orientan una comunicación abierta a los sentidos, por lo que presentan una lectura siempre cambiante.

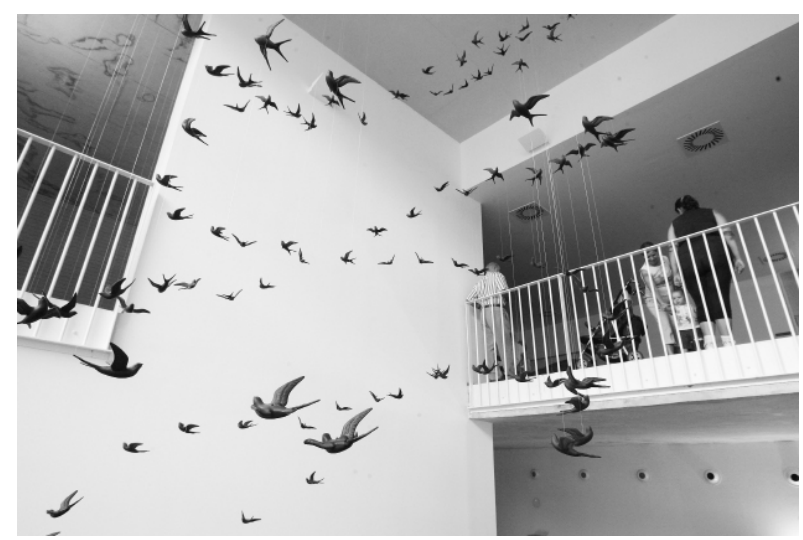

Fig.3. Pamen Pereira. El ADN del amor. Hall consultas externas.

En total, son siete artistas los que han trabajado las obras escultóricas que integran este proyecto. Ubicadas tanto en el exterior como en el interior del edificio, han sido encargadas a Irma Ortega y Felipe Ferrer, autores de la obra "Sello: Sellando la Salud"; José Antonio Orts con su propuesta "Pulsaciones"; Carmen Marcos y Carlos Martínez Barragán con "Espacio Poético", Pamen Pereira, autora de "ADN, los órdenes del amor"; Joan Llaveria, con dos propuestas bajo el título de "ECOSISTEMAS". 
Obras que buscan desde invitar al espectador a la reflexión y al optimismo, a señalar el pulso vital, a evocar el flujo del tiempo y de la vida, o a mostrar simbólicamente la molécula protagonista de nuestra biología, el ADN.

\section{Pinturas Murales: un diseño de ensoñación para Pediatría}

Un proyecto mural desarrollado por Sr. Marmota, Julieta, Deih e Yñigo, cuatro de los ocho miembros de la crew XLF, un destacado colectivo del Urban Art valenciano, es la manifestación artística elegida para todos los espacios del hospital pertenecientes al área de Pediatría.

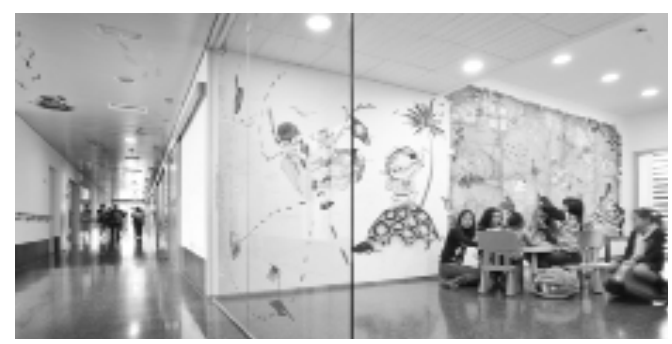

Fig. 4. Pasillo y sala de juegos de pediatría. XLF Crew

Una iniciativa que también ha sido desarrollada conjuntamente entre el Hospital y el Instituto de Arte y Entorno de la UPV.

Así, esta muestra de Urban Art impregna desde la sala de espera de Urgencias de Pediatría hasta la sala de juegos de planta, pasando por las 11 habitaciones reservadas para los más pequeños, que muestran cada una de ellas un motivo y lenguaje pictóricos diferente.

Los artistas que han llevado a cabo esta obra han contado con la ayuda de los responsables de los distintos equipos pediátricos, y juntos han definido los contenidos de los murales plasmando mensajes relacionados con la importancia de la lectura, el deporte, la higiene o la alimentación sana.

El pop art, el manga japonés y una serie de códigos icónicos han servido de inspiración para esta intervención plástica que no ha dejado ni un espacio sin pintar, incluidos los techos, el primer lugar que miran los niños al salir de quirófano.

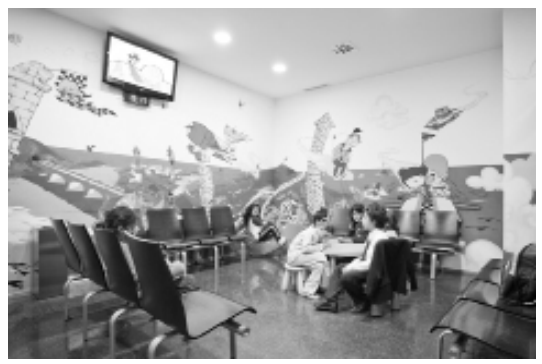

Fig.5 Sala de urgencias pediátricas. XLF Crew 
Con este trabajo se busca mejorar la sensación de confort, a través de unos colores que facilitan la relajación y contribuyen al bienestar de los pacientes, favoreciendo su recuperación. Y es que física y anímicamente el ser humano se siente influido por los colores y las imágenes que le rodean.

La naturaleza y el imaginario propio de cada uno de los cuatro artistas y su bagaje iconográfico han servido de leitmotiv para abordar las diferentes paredes. Imágenes con las que se busca la interacción con los niños, despertando su imaginación, favoreciendo el diálogo y el intercambio de sueños.

\section{Sala de Exposiciones del proyecto cuidArt}

Ubicada en la planta baja del hospital, esta sala está diseñada para una media prevista de seis a cuatro exposiciones al año. La Sala cuidArt acoger muestras que vinculen el tema del Arte y la Salud. Estas exposiciones, de carácter temático, serán tratadas en ocasiones por colectivos artísticos y en otras por artistas individuales y siempre en colaboración con algún o algunos servicios sanitarios del Hospital.

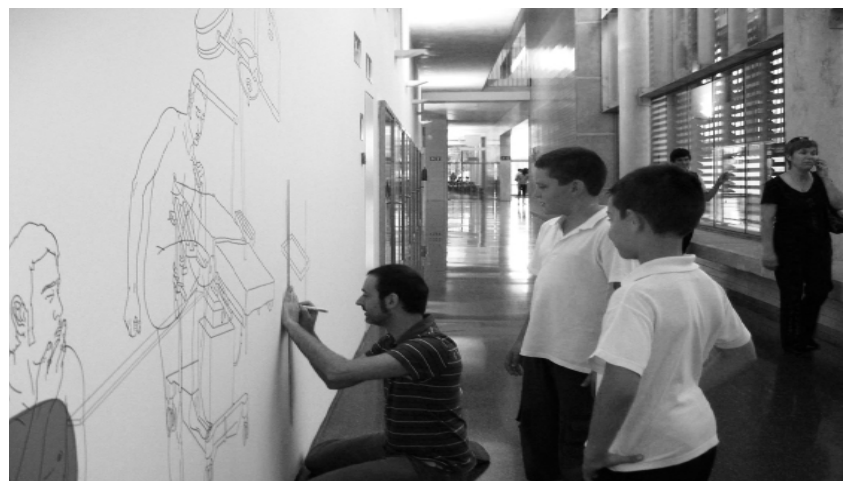

Fig. 6 Moisés Mahiques trabajando su intervención mural en la sala cuidArt

Hasta la fecha se ha mostrado la obra de: Moisés Mahiques: Alter a sweet illness. Juan Olivares: Píldoras Visuales. Gervasio Sánchez: Vidas Minadas. Fontilles, 100 años trabajando por un mundo sin lepra. Exposición de trabajos realizados por pacientes a lo largo de los talleres de 2010. Rosalía Banet: Para comerte mejor. Melli Pérez-Madero: Agua. Reflexiones sobre la necesidad del agua en el cuerpo humano. (en colaboración con el departamento Anestesia).

\section{Arte en vivo}

Parte fundamental de este programa de actividades para el Hospital de día en las áreas de oncología y hemodiálisis son las intervenciones artísticas en tiempo real que mensualmente los artistas comparten con los pacientes que pasan el mayor tiempo en 
esta sala durante su proceso de recuperación. Diferentes colectivos y autores practican arte en vivo: intervenciones murales efímeras, cuadros en tiempo real, esculturas. El resultado es arte en mayúsculas realizado con la participación de los pacientes que vuelcan su imaginario en los muros que los envuelven, dando así lugar a liberar sentimientos, preocupaciones y alegrías.
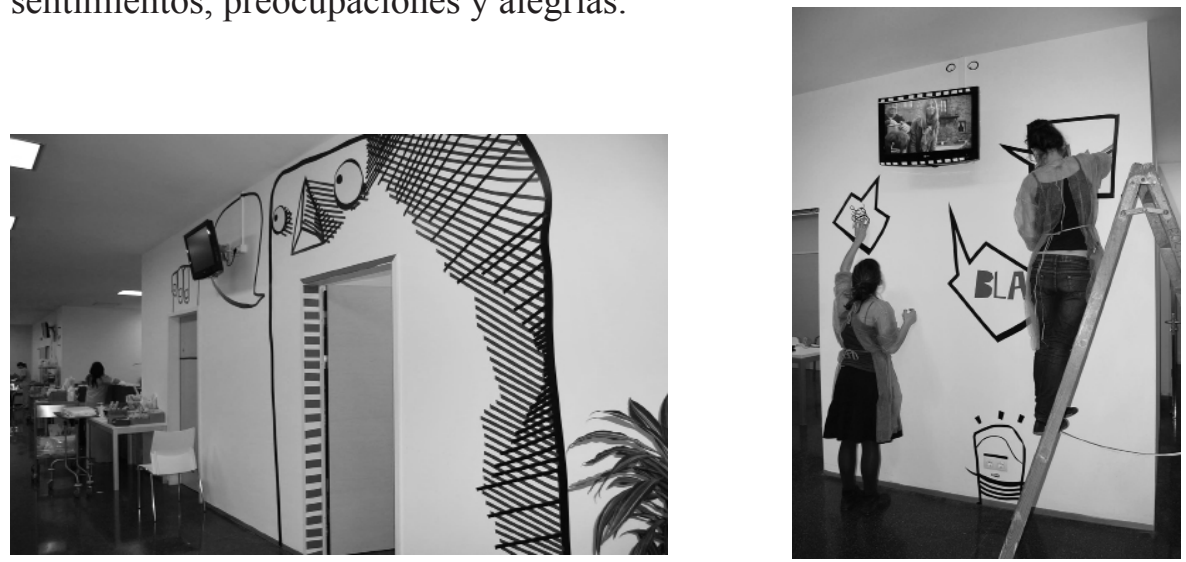

Fig. 7 y 8. Colectivo VIRA-LATA trabajando en su ARTE EN VIVO en la sala de Hemodiálisis.

\section{Talleres para pacientes}

Recientemente se ha incorporado al proyecto cuidArt un programa de talleres de distintos ámbitos para pacientes de las distintas áreas del hospital. Estos talleres se llevan a cabo por arteterapeutas de diferentes disciplinas: artes plásticas, música, teatro. Existen investigaciones en el mundo que dan cuenta de los beneficios que ofrece la participación de niños y adultos en experiencias artísticas (Dileo, 2009) (Geue, 2010), las cuales promueven, entre otras cosas, mejoras en su sistema inmunológico, su autoestima, socialización y capacidad de afrontamiento. Trabajar estas capacidades, es muy importantes para los pacientes (e incluso para el personal sanitario), quienes al verse aislados de su ambiente familiar y social adquieren cierta vulnerabilidad, que sumada a la situación clínica que padecen, genera en el universo del niño o adulto un conflicto ante el que es difícil permanecer indiferente. Trabajar con las potencialidades y capacidades del individuo es vital para que su organismo responda de un modo más favorable ante la situación de vulnerabilidad en que la enfermedad lo ha colocado, y para conseguir una mejor respuesta emocional ante esta situación (Geue, 2010).

El personal sanitario es convocado con una frecuencia de tres o cuatro veces al año para realizar talleres que van desde la literatura a las artes plásticas. Además se beneficia de los talleres realizados a los pacientes. Su presencia y participación se convierte vital en la observación de las terapias y mediciones de las mismas, así como evaluaciones e informes pertinentes que se vuelcan con una frecuencia bimensual en reuniones entre el personal sanitario, la dirección de investigación del hospital y los monitores 


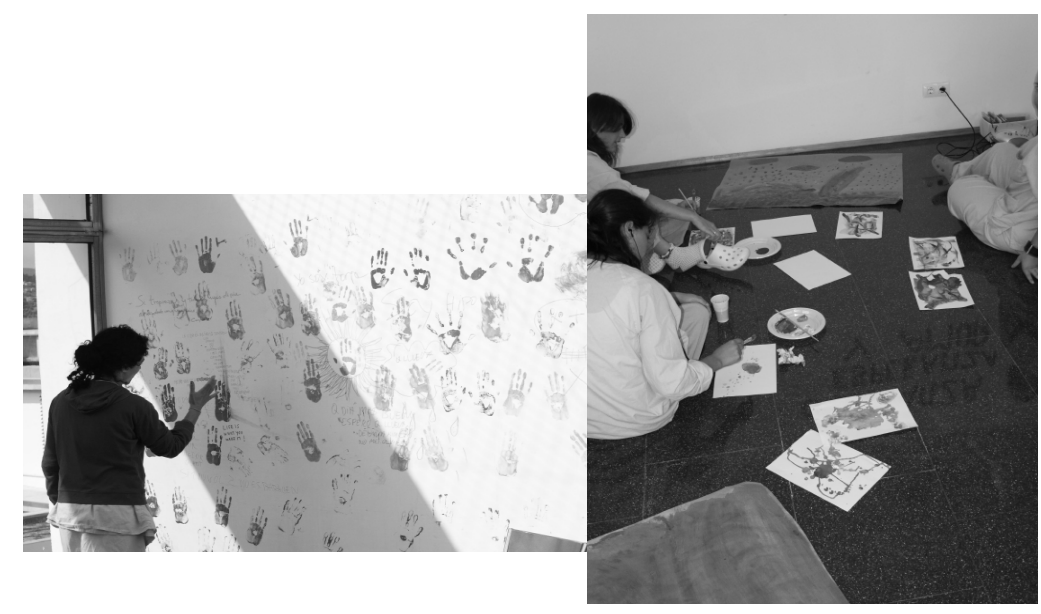

Fig.8 y 9 Talleres de arteterapia en el área de psiquiatría

\subsection{Talleres arteterapia impartidos por Isabel Soler}

Empezamos los talleres de artes plásticas en mayo de 2010. Las primeras sesiones se realizaron en los Servicios de psiquiatría y de pediatría, y se plantearon como "talleres ocupacionales". Durante las sesiones se utiliza fundamentalmente la plástica, aunque resulta importante utilizar otras disciplinas a modo de soporte como la escritura, el trabajo corporal o la música.

Con la ayuda de unos desencadenantes de implicación personal, el individuo se proyecta en las obras que realiza y se incluye emocionalmente en la creación aún sin saberlo. El arte permite expresar sentimientos y emociones que son muy difíciles de expresar verbalmente, y dicha expresión permite sublimar miedos, frustraciones y angustias. En un ambiente de libertad, las obras producidas se convierten en terapéuticas al lograr eludir los mecanismos de defensa y las estructuras rígidas, la persona se expresa a través de sus creaciones y recorre un camino acompañada por el/la terapeuta.

En psiquiatría las sesiones se llevan a cabo un día a la semana durante una hora y media. Las personas ingresadas en este Servicio suelen llegar en situación de crisis y permanecen en el Centro un periodo corto de tiempo en el que se les diagnostica, estabiliza y controla la medicación. Al no ser posible un seguimiento de los pacientes, las actividades se centran en activar sentimientos y emociones positivas para mejorar la actitud del paciente ante su dolencia.

La satisfacción entre pacientes y acompañantes y entre los profesionales sanitarios ha permitido extender las actividades al Servicio de Oncología.

En oncología se realizan sesiones de una hora por la mañana, generalmente antes de entrar en la sesión de quimioterapia. Esta intervención se halla en una fase de implantación progresiva, con muy buenos resultados en los pacientes que decidieron participar. El desconocimiento de este tipo de técnicas por los pacientes ha sido 
probablemente uno de los impedimentos para una mayor participación, y se espera corregir esta situación con mayor y mejor información sobre la actividad.

Otra actividad que se está realizando es la proyección de documentales en la sala de quimioterapia y hemodiálisis. Estos documentales están relacionados con el arte y la naturaleza y se seleccionan de forma que resulten atractivos tanto a nivel estético como de contenido. La finalidad es que despierten interés y ayuden a mantener la atención en algo más allá de la enfermedad.

\subsection{Proyecto de intervención en Musicoterapia en el Hospital de Dénia. Impartido por Ana Alegre - Isabel Bellver - Maria Selva Lodeiro}

La atención que se está brindando desde marzo del presente año a los pacientes del Hospital de Dénia, viene determinada por la delimitación de los casos y las necesidades que desde la propia institución se han evidenciado, y que se ajustan al tipo de intervención que realizan los musicoterapeutas:

- Favorecer la expresión de emociones.

- Estimular la comunicación.

- Favorecer la aceptación de la situación de ingreso hospitalario.

El proyecto de intervención en Musicoterapia de este Hospital se ha estructurado en función de diversas poblaciones objetivo, atendidas en cuatro Unidades: Neonatología, Psiquiatría, Hemodiálisis y Oncología (Hospital de Día).

Es importante destacar que las intervenciones musicoterapéuticas son diseñadas específicamente para cada una las Unidades mencionadas.

La música y las actividades musicoterapéuticas empleadas atienden claramente a dos áreas de intervención bien definidas, las relacionadas con necesidades físicas, o bien vinculadas a necesidades emocionales y psicosociales:

- Favoreciendo habilidades de afrontamiento.

- Elevando el estado de ánimo.

- Reduciendo la ansiedad.

- Aumentando la adherencia terapéutica.

- Normalizando el entorno.

Las técnicas usadas incluyen, por una parte, la implicación de los pacientes y/o familiares, cuando sus circunstancias lo permiten, en actividades expresivas y activas, mediante el uso de diferentes elementos sonoros, tales como instrumentos y la propia voz. Y por otra parte, se utilizan técnicas de tipo receptivo, en que pacientes y familiares son implicados en las actividades mediante la escucha y percepción de estímulos sonoros, que provienen de música interpretada en vivo por las musicoterapeutas.

La intervención se realiza en la sala y con el consentimiento del paciente o de los familiares.

El proceso de intervención en Musicoterapia que se está desarrollando en el Hospital de Dénia incluye diferentes fases de actuación:

- Evaluación inicial y delimitación de objetivos.

- Elaboración y puesta en marcha del plan de intervención.

- Evaluación continua y ajustes de objetivos y recursos.

- Evaluación final y propuesta de seguimiento. 
El equipo está formado por tres musicoterapeutas: Ana Alegre e Isabel Bellver, que realizan atención directa una vez por semana en las cuatro unidades anteriormente mencionadas, y María Selva Lodeiro, que realiza labores de supervisión y dirección del proyecto.

Dicho proyecto, pionero en la Comunidad Valenciana, se ha iniciado en marzo de 2011 y tiene una duración prevista de 6 meses.

Según los datos obtenidos hasta la fecha, se atienden a una media de 25 pacientes semanalmente.

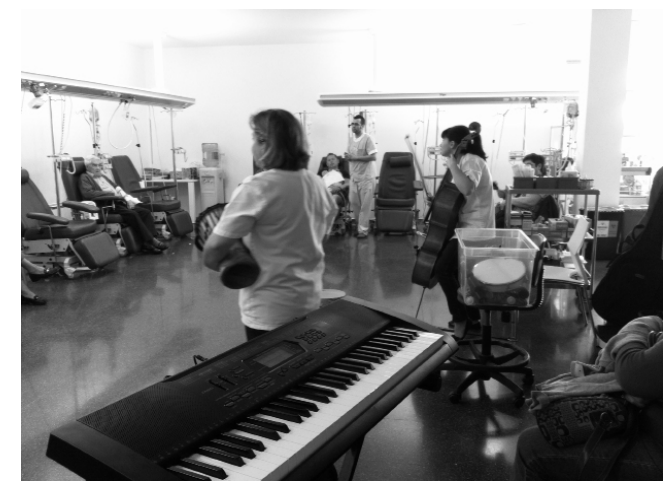

Fig.10. Taller de musicoterapia en el Hospital de Día

La experiencia realizada hasta este momento está resultando muy positiva. Los primeros resultados obtenidos en el estudio experimental llevado a cabo por el equipo de musicoterapeutas, deja entrever una acogida muy buena tanto por parte de los pacientes y sus familiares, como por parte del equipo médico de las diferentes unidades.

La metodología empleada para este estudio consta de cuestionarios aplicados antes y después de las sesiones, encuestas de satisfacción y grabaciones en vídeo.

Un primer análisis de la información recogida ha permitido un mayor conocimiento del trabajo realizado por los musicoterapeutas por parte del equipo médico, que se ha traducido en un mayor apoyo para introducir la musicoterapia como terapia complementaria del tratamiento estándar ofrecido habitualmente a los pacientes del hospital, con lo que la intervención musicoterapéutica se torna una herramienta eficaz que se utiliza en beneficio del paciente.

Podemos decir que la musicoterapia está generando en los participantes y familiares una respuesta muy buena, pudiendo constatar su motivación durante las sesiones y su satisfacción con el proyecto desarrollado hasta el momento.

\section{Colaboraciones con la comarca y las universidades: un mundo de posibilida- des al servicio del arte}

La última apuesta del proyecto cuidArt es un plan de colaboraciones con otras instituciones públicas y privadas para compartir e intercambiar experiencias artísticas y de investigación. 
Entre ellas, cabe destacar la Cátedra DKV Arte y Salud con la Facultad de Bellas Artes de la Universidad Politécnica de Valencia. En ella se desarrollan diferentes becas de apoyo a jóvenes artistas así como el concurso Fresh Art, dirigido a estudiantes de bachillerato artístico. Se están iniciando las primeras investigaciones acerca de humanización de hospitales, entornos sanitarios y temas relacionados con el arte y la salud. Estas investigaciones se pretenden que sean volcadas en un congreso bianual dirigido al ámbito de la medicina, psicología, bellas artes, diseño, arquitectura y todos aquellos agentes que puedan intervenir.

Otra de las grandes iniciativas de cuidArt dentro de su programa de Colaboraciones, es el concurso fotográfico de lactancia materna, impulsado por el Grup Nodrissa, integrado en su mayoría por el personal de Pediatría. En colaboración con el servicio de maternidad y obstetricia y el citado grupo, se han seleccionado unas 40 fotografías que decoraran las salas de estos servicios. Una muestra que recorre desde las salas de dilatación, lactancia materna, neonatos o en los propios pasillos de maternidad.

La siguiente via de trabajo dentro del compromiso de Colaboraciones de cuidArt es la cesión de las exposiciones vistas en el Hospital a los ayuntamientos de la zona de la Marina Alta. Acercamos el arte a los ciudadanos. No solo se trata de la gestión del Hospital, sino de todo el departamento de salud de Dénia.

Asimismo cada año se organiza desde el hospital, en colaboración con los Ayuntamientos de la Marina Alta, el Concurso nacional de fotografía Cuida't Vida Sana, que promueve las costumbres saludables. Posteriormente se produce una exposición que se inaugura en el hospital para luego itinerar por todas las salas de los ayuntamientos colaboradores.

\section{Medición de resultados y proyectos de investigación}

Actualmente no existe ninguna descripción en la literatura revisada sobre una intervención múltiple como la que se lleva a cabo en el Hospital de Dénia. Por tanto, resulta difícil anticipar el efecto sobre los pacientes y acompañantes expuestos a las intervenciones del programa. Tampoco es posible estimar a priori el efecto del programa sobre los observadores que no participan directamente, incluyendo al personal sanitario y no sanitario que trabaja en el Hospital.

El objetivo actualmente es realizar mediciones cuantitativas de los efectos de las intervenciones en cada una de las personas implicadas. Para ello, se ha diseñado un programa complejo que tiene en cuenta las características específicas de cada tipo de intervención y cada tipo de participante, con el resultado de al menos doce diseños de investigación distintos.

Los ejes de medición fundamentales incluyen las siguientes variables:

Calidad de vida, según escalas generales [EuroQuoL-5D-5L (Rabin, 2011) o SF-36 (Vilagut, 2005)], y adaptadas a patologías concretas [EORTC QLQ-C30 (Aaronson, 1993) para pacientes oncológicos, o KDQOL (Hays, 1997) para pacientes en Hemodiálisis].

Efectos sobre la salud mental de los pacientes, según escalas validadas de medición de ansiedad [STAI (Chlan, 2003)], depresión [Inventario de depresión de Beck (Sanz, 1998)], o autoestima [escala de autoestima de Rosenberg (Vázquez, 2004)], y 
efectos somáticos de las intervenciones (variación de frecuencia cardiaca y respiratoria, tensión arterial).

Satisfacción con las intervenciones. Para ello se trabajará con escalas de satisfacción genéricas, adaptándolas a las características de cada método y de su población objetivo.

Desde el comienzo del programa de intervenciones se planteó que un cuarto eje fundamental para el éxito del programa sería la coordinación con la Dirección y los trabajadores del Hospital (Delamothe, 1989). Para ello han resultado fundamentales el flujo constante de información y el trabajo sin interferencias mutuas.

Aunque se ha comprobado informalmente la satisfacción de la mayoría de los trabajadores sanitarios con los efectos de las distintas intervenciones sobre los pacientes y ellos mismos, se ha decidido diseñar una encuesta que explore sistemáticamente las dimensiones críticas de esta interacción, dado que puede presentar beneficios emocionales para los trabajadores (Theorell, 2009). Esta encuesta se basa en el marco teórico generado a través de la investigación cualitativa en esta área (O'Callaghan, 2009).

Actualmente se han realizado mediciones basales en la mayoría de los pacientes en los que se espera una intervención constante durante un periodo de seguimiento de al menos 6 meses. Este es el caso de Hemodiálisis y algunos de los pacientes de Oncología. El largo seguimiento de estos pacientes y la presencia de grupos de control en Hemodiálisis hace que valga la pena el esfuerzo que supone para el paciente cumplimentar escalas muy exhaustivas, ya que la comparación con los resultados tras 6 meses de intervención puede enriquecer considerablemente la valoración de las intervenciones, y ofrecer una base cuantitativa para su adaptación a la población objetivo.

En el caso de pacientes en los que se espera realizar únicamente intervenciones puntuales, se están diseñando cuestionarios que incorporen escalas validadas fáciles de contestar [por ejemplo, una versión abreviada del cuestionario STAI (Chlan, 2003)], con un tiempo de respuesta total de no más de 5 minutos, para su cumplimentación antes y después de cada intervención. Este es el caso de los pacientes de Psiquiatría, Pediatría, y los que acuden al Hospital de Día Médico. Aunque la falta de seguimiento limita la evaluación al efecto a corto plazo, esto supone una base cuantitativa para valorar, comparar y adaptar las intervenciones a estos colectivos.

En todos los casos se ha seleccionado a terapeutas con experiencia en el ámbito sanitario, pero la especial complejidad de algunos grupos de pacientes ha hecho necesario definir un período de adaptación previo a las mediciones, para evitar el sesgo que supondría la recogida de datos en un período que se caracterizará por un primer ajuste a las necesidades de cada tipo de paciente. Pasado este periodo, y sólo cuando los terapeutas hayan optimizado su sistemática de trabajo, comenzarán las mediciones a todos los niveles.

Quedan pendientes de establecer los efectos de las intervenciones con métodos de investigación cualitativa. Estas técnicas resultarán fundamentales para complementar algunas de las deficiencias inherentes a los análisis cuantitativos, y para captar toda la riqueza de efectos de las intervenciones en curso (Íñiguez, 1999) (Edwards, 2005). Para ello es necesario que se incorpore al grupo al menos un investigador con 
experiencia en técnicas cualitativas, que se encargue de dirigir el trabajo de campo e interpretar los resultados. Actualmente, sólo el equipo de musicoterapia realiza sistemáticamente este tipo de evaluación, utilizándola para adaptar sus intervenciones de forma continua y valorar los efectos obtenidos con cada cambio.

Los efectos a largo plazo de las intervenciones se pondrán en relación con los costes asociados, por medio de análisis de coste-efectividad. Por último, en aquellos casos en los que existan mediciones del cambio en calidad de vida, se realizará un análisis coste-utilidad que permita cuantificar el coste por año de vida ajustado por calidad. Este análisis económico resulta fundamental para valorar la eficiencia y viabilidad de este tipo de intervenciones en un entorno con recursos limitados, dado que no existen estudios de este tipo entre la literatura revisada.

\section{Referencias}

Aaronson, N.K, Ahmedzai, S., Bergman, B., Bullinger, M., Cull, A., Duez, N.J. et al. 1993: The European Organisation for Research and Treatment of Cancer QLQ-C30: A quality-of-life instrument for use in international clinical trials in oncology. Journal of the National Cancer Institut, 85, 365-376.

Belver, M.H., Ullán, A.M. 2010: Symbolic environmental mediators in health settings: the role of art in the humanization of children's hospitals. Arte, Individuo y Sociedad,22(2), 73-81.

Campoy, L.F., Santacreu, J. 2008: La colaboración público-privada en el marco del Sistema Nacional de Salud. El caso especial del modelo valenciano a propósito de la concesión de Denia. Revista de Administración Sanitaria, 6(2), 297-321.

Chlan, L., Savik, K., Weinert, C. 2003: Development of a shortened state anxiety scale from the Spielberger State-Trait Anxiety Inventory (STAI) for patients receiving mechanical ventilatory support. Journal of nursing measurement, 11(3), 283-293.

Clift, S., Camic, P.M., Chapman, B., Clayton, G., Daykin, N., Eades, G. et al. 2009:

The state of arts and health in England. Arts \& Health, 1(1), 6-35.

Delamothe, T. 1989: Hospital art and its problems. BMJ, 298, 1164-5.

Dileo, C., Bradt, J. 2009: On creating the discipline, profession, and evidence in the field of arts and healthcare. Arts \& Health, 1(2),168-182.

Edwards, J. 2005: Possibilities and problems for evidence-based practice in music therapy. The arts in psychotherapy, 32, 293-301.

Geue, K., Goetze, H., Buttstaedt, M., Kleinert, E., Richter, D., Singer, S. 2010: An overview of art therapy interventions for cancer patients and the results of research. Complementary therapies in medicine, 18, 160-170.

Hays, R.B., Kallich, J.D., Mapes, D.L., Coons, S.J., Amin, N., Carter, W.B. et al. 1997: Kidney Disease Quality of Life Short Form (KDQOL-SFtm), Version 1.3: A manual for use and scoring. RAND,. Disponible en: http://gim.med. ucla.edu/kdqol/downloads/kdqol13man.pdf 
Íñiguez, L. 1999: Investigación y evaluación cualitativa: bases teóricas y conceptuales. Atención Primaria, 23(18), 496-502.

Lawson, B. 2010: Healing architecture. Arts \& Health,2(2), 95-108.

O'Callaghan C, Magill L. 2009: Effect of music therapy on oncologic staff bystanders: a substantive grounded theory. Palliative \& Supportive Care, 7(2), 21928.

Rabin, R., Oemar, M., Oppe, M., Janssen, B., Herdman, M., 2011: en representación del Grupo EuroQol. EQ-5D-5L User Guide. Versión 1.0. Disponible en: http:// www.euroqol.org.

Sanz, J., Vázquez, C. 1998: Fiabilidad, validez y datos normativos del inventario para la depresión de Beck. Psicothema, 10(2),303-318.

Sonke, J., Rollins, J., Brandman, R., Graham-Pole, J. 2009: The state of the arts in healthcare in the United States. Arts \& Health, 1(2),107-135.

Theorell, T., Hartzell, M., Näslund, S. 2009: A note on designing evaluations of health effects of cultural activities at work. Arts \& Health,1(1), 89-92.

Vázquez, J., Jiménez, R., Vázquez-Morejón, R. 2004: Escala de autoestima de Rosenberg: fiabilidad y validez en población clínica española. Apuntes de Psicología, 22(2),247-255.

Vilagut, G., Ferrer, M., Rajmil, L., Rebollo, P., Permanyer-Miralda, G., Quintana, J.M. et al. 2005: El cuestionario de salud SF-36 español: una década de experiencia y nuevos desarrollos. Gaceta Sanitaria,19(2),135-50. 